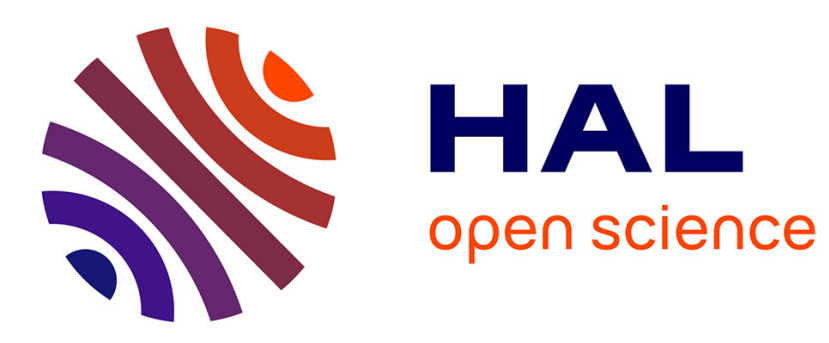

\title{
Magnetomechanical Damping at Low Temperatures
}

D. Beshers

\section{To cite this version:}

D. Beshers. Magnetomechanical Damping at Low Temperatures. Journal de Physique IV Proceedings, 1996, 06 (C8), pp.C8-523-C8-526. 10.1051/jp4:19968113 . jpa-00254543

\section{HAL Id: jpa-00254543 https://hal.science/jpa-00254543}

Submitted on 1 Jan 1996

HAL is a multi-disciplinary open access archive for the deposit and dissemination of scientific research documents, whether they are published or not. The documents may come from teaching and research institutions in France or abroad, or from public or private research centers.
L'archive ouverte pluridisciplinaire HAL, est destinée au dépôt et à la diffusion de documents scientifiques de niveau recherche, publiés ou non, émanant des établissements d'enseignement et de recherche français ou étrangers, des laboratoires publics ou privés. 


\title{
Magnetomechanical Damping at Low Temperatures
}

\author{
D.N. Beshers \\ Henry Krumb School of Mines, Columbia University New York, NY 10027, U.S.A.
}

\begin{abstract}
Magnetomechanical damping (MMD) at low temperatures shows marked departures from the well-established phenomenological description of MMD at room temperature. There is enhanced damping (the Bruner-Heller rise) at low amplitudes generally said to have a magnetic origin, be hysteretic, and sometimes amplitude-dependent, but the falloff in the transition to higher temperatures is frequency-dependent. A recent model considers that the Rayleigh MMD at room temperature arises from successive cooperative shifts (self-similar transformations) of the whole domain structure at once. It appears that the Bruner-Heller rise in pure iron may well be a nanifestation of the localized hysteretic bowing motion of donain walls, the equivalent of the Granato-Lücke danping for dislocations. For alloys, the data are supportive but incomplete.
\end{abstract}

\section{INTRODUCTION}

The standard description of magnetomechanical damping (MMD) includes a hysteretic part that shows a linear rise with strain amplitude over a wide range. In iron this region, often called the Rayleigh region, runs from about $10^{-6}$ to $10^{-4}$ in strain amplitude. The salient, indeed the only, feature of the Rayleigh region is its slope, $\mathrm{dQ}^{-1} / \mathrm{d} \epsilon=\theta_{\mathrm{R}}$. With respect to plastic deformation, $\theta_{\mathrm{R}}$ is largest in annealed specimens, lower in deformed specimens. The damping then is attributed to the motion of domain walls, walls which move more freely when there are no obstacles. The phenomenological theory of Smith and Birchak [1,2] (SBI and SBII) -- which assumes a distribution of local values of internal stress about some average value $\sigma_{i}$ but does not specify the spatial distribution -- predicts that the slope is proportional to $\sigma_{i}^{-2}$, a prediction confirmed for the case of cold-worked specimens. [3]

In recent years it has been recognized, and stated with increasing forcefulness [3-5], that the linear rise of the Rayleigh region is a power-law behavior without any physical scale, and so there may be some self-similar, or fractal, curve underlying the dissipative process. The situation for domain walls contrasts strikingly with that for dislocations [4] which most definitely show transitions as the strain amplitude is increased and thereby show scales of stress, and therefore of length. A detailed argument of the case appeared recently [5].

The possibility of a self-similar aspect of domain wall motion was suggested by Vergne, Porteseil, and Cotillard (VPC) [6] after observing the flux variations accompanying the motion of a 180" domain wall moving at constant velocity; their experiment was not mechanical but we return to it below for important confirmation. Power laws with scaling had already been put forward by Hilzinger [7] as part of a general theory of the motion of linear and planar structures through randomly distributed fields of obstacles. The essential feature of Hilzinger's scaling theory of domain wall motion is the random distribution of obstacles, and the self-similar motion of a domain wall observed by VPC is likewise concerned with a spatial distribution of obstacles; these approaches are candidates to explain the Rayleigh region. However, some of the data to be considered are on iron of high purity in the annealed condition, which seems to point toward a mechanism that depends only on the presence of the domain structure, not on other defects. Another general suggestion, important but undeveloped in this context, was that of topological hysteresis put forward by Molho and Porteseil [8], in which the nucleation of new domain walls is the key process; such a mechanism clearly involves only the domain structure.

Lately [5] we have suggested a mechanism for magnetomechanical damping (MMD) that depends only on transitions between discrete domain structures and so requires no other defect to be present. 
That suggestion satisfies the need [4] for a collective mechanism, as opposed to an individual particle mechanism, but leaves open the question of what place to give to individual particle mechanisms, by which we mean those that can be modeled by a sort of two-body interaction between a domain wall and some crystal defect. The experiments on which VPC [6] based their suggestion of fractal behavior clearly revealed the sort of situation that Hilzinger [7] had in mind; those experiments were at low temperatures. Indeed, the standard description of MMD is based largely on data at room temperature, or above. The MMD data at low temperatures presented below have a very different form; taking these together with some data on MMD in iron alloys, leads us to a new synthesis consistent with VPC and Hilzinger.

\section{THE EXPERIMENTS OF INTEREST}

\subsection{Magnetomechanical Damping}

The available data on MMD in iron at low temperatures include only two reports on amplitude dependence, those of Guberman and Beshers (GBI) [8] and Govaer and Beshers (GBII) [9]. A detailed comparison was given by GBII. All agree that there is a rise in damping at low temperatures (the Bruner-Heller rise) thought to be of magnetic origin, but only GBII actually demonstrated that the rise is suppressed by a magnetic field. The collected data show that at lower frequencies the rise occurs at lower temperatures. There appears to be a different hysteretic process at low temperatures, at least one, a process that can be speeded by thermal activation; when the temperature is high enough the barriers are no longer effective.

The studies of amplitude dependence at low temperatures by GBI and GBII used zone-refined iron, and the purity of the material is significant for us. Indeed only GBI and GBII reported amplitude dependence, but most of the specimens of other workers were heavily hydrogenated. Figure 1, taken from GBI, show damping versus amplitude, at $32 \mathrm{kHz}$ and zero field, from room temperature down to $82 \mathrm{~K}$. The initial rise at $297 \mathrm{~K}$ has a $\theta_{\mathrm{R}}$ of 25 , comparable with other observations at frequencies from $17 \mathrm{~Hz}$ to $50 \mathrm{kHz}$ [3]. There is a marked change of behavior as the temperature decreases: damping in the Rayleigh region falls with the ascending straight line replaced by a descending curve. Data at still lower amplitudes reveal an actual peak in damping versus amplitude about $\epsilon=10^{-6}$ as may be seen in Figure 3 of GBII.
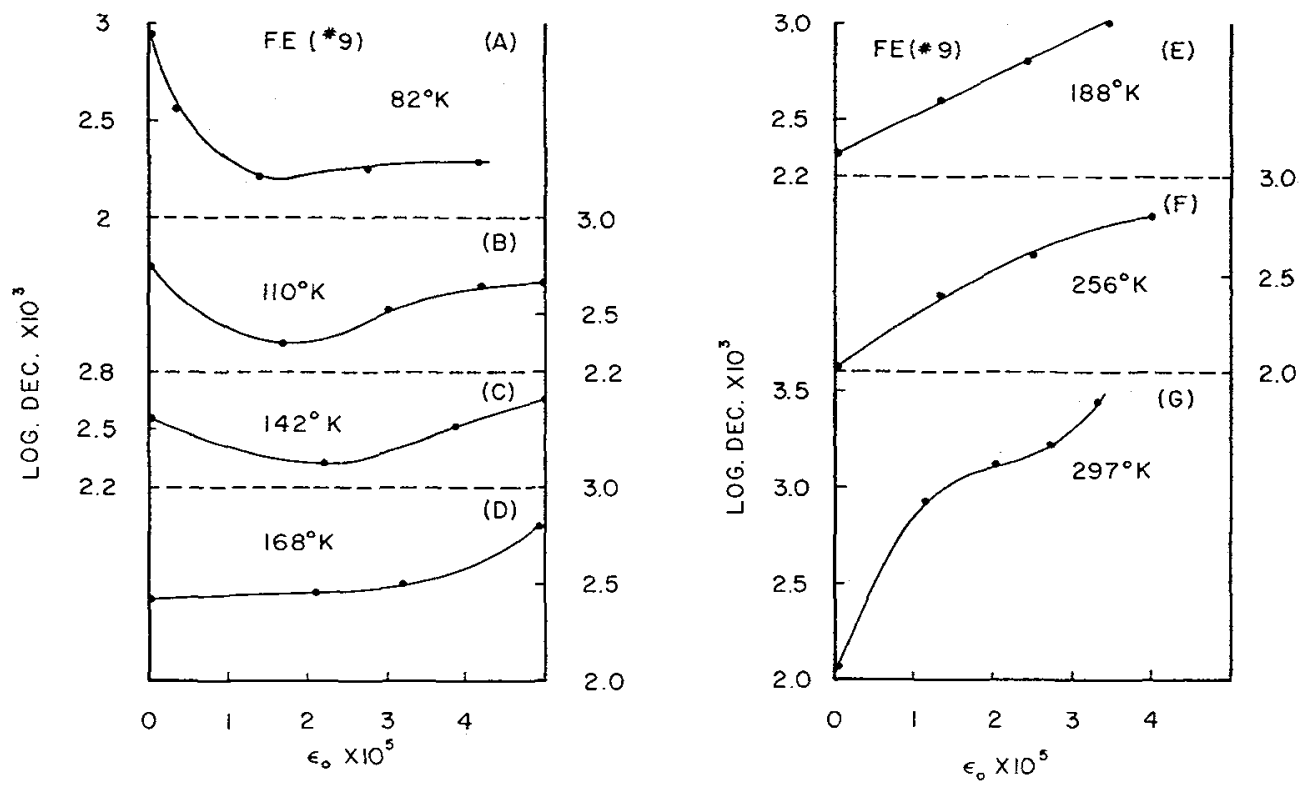

Figure 1: Amplitude dependence of zone-refined iron from room temperature down to liquid nitrogen temperature with no magnetic field. The logarithmic decrement $\Delta=\pi \mathrm{Q}^{-1}, \epsilon_{0}$ is strain amplitude. From [8]. 


\subsection{Iron alloys}

Smith and Birchak (SB III) [10] and Birchak and Smith (SBIV) [11] have reported data on the hysteretic MMD in FeGe and FeSi alloys at room temperature. They find values of $\theta_{\mathrm{R}}$ that range up to about 2400 in $\mathrm{FeSi}$ annealed at $1200^{\circ} \mathrm{C}$, more than 100 times the slope shown in curve (G) of Fig. 1, corresponding to $\sigma_{\mathrm{i}}=2 \mathrm{MPa}$; if this internal stress is attributed all to dislocations their density would be about $5 \times 10^{11} \mathrm{~m}^{-}$ ${ }^{2}$, a reasonable value for a well-annealed specimen. However, once we have made that attribution there is nothing left over for other sources of internal stress, that is there is no sign of solid solution hardening for the domain walls!

\subsection{Domain wall dynamics}

Evidence supporting a transition in dynamics of domain walls at lower temperatures is found in the experiments of Porteseil et al. [12] With a crystal of FeSi in the shape of a picture frame, they observed the motion of a single macroscopic $180^{\circ}$ wall that formed a closed loop around the frame. From 10 to $50 \mathrm{~K}$ they observed a magnetic creep in response to small steps of field. From 253 to $323 \mathrm{~K}$ the wall was forced to move at constant velocity by a magnetic field controlled by feedback from a pickup coil. The fluctuations in the signal from the pickup coil represented jumps in the domain wall. The creep at lower temperatures was thought to involve the same mechanism as the fluctuations around $250 \mathrm{~K}$. The fluctuation curve was found to be self-similar, i.e. fractal, with characteristic volume of $10^{-7} \mathrm{~cm}^{3}$ at 283 $\mathrm{K}$, varying as $\mathrm{T}^{4.4}$; this volume corresponds to a linear size of about $50 \mu \mathrm{m}$. In a polycrystalline specimen, coherent motion of a single wall is not possible at scales of the order of the grain size. The results of Porteseil et al. suggest that one should see a change in damping mechanism, at temperatures depending on the frequency, in the range at which the Bruner-Heller rise is replaced by the Rayleigh law.

\section{SYNTHESIS}

The situation is that the MMD at room temperature in both pure iron and in the silicon and germanium alloys follows a Rayleigh law with no physical scale such as length or stress. At low temperatures the pure iron shows evidence of such scales. This suggests that some obstacles were present despite the purity but only at low temperature were they effective pins; the damping with pinning may then be analogous to the Granato-Lucke dislocation damping and we may suppose the pins to be fairly dilute.

There is no low temperature damping data for the alloys, but there are the observations [12] of fluctuations of a moving domain wall in a crystal of FeSi. In contrast to the situation for pure iron, these fluctuations indicate [6] that the wall was moving through a field of obstacles with self-similar aspects to their distribution and strength, that is without scale. The same observations indicate that the individual jumps increase in size with temperature, to the point where they become larger than the domain size in ordinary polycrystalline specimens; there is probably a change in damping mechanism when that happens because the obstacles are no longer able to restrain the domain walls effectively. Indeed, the room temperature MMD, much larger for the alloys than for the pure iron, shows no sign of solid solution hardening of the magnetic aspects.

These observations are consistent with a picture of room temperature damping arising from a mechanism intrinsic to the domain structure, insensitive to the obstacles raised by fluctuations in the alloy distribution, but still affected by the dislocation structure of cold-work. The compositions involved here were such that the $\alpha-\gamma$ transition was suppressed so the specimens could be annealed at quite high temperatures without damage from a phase transformation with an appreciable volume change. Indeed, $\theta_{\mathrm{R}}$ increased substantially with annealing temperature [11] as would be expected on this picture.

If this picture is correct, then whatever self-similar aspect the room temperature damping may have, it is not that suggested by Hilzinger [7] and derived by VPC [6\} from observations. In any case the damping is present in pure iron, and must be explained for that important special case.

It was pointed out recently [5] that the domain structure exists because it reduces the external field of a body and thereby reduces the magnetic energy. The ideal domain structure eliminates all external field, at the cost of the energy of the domain walls themselves. There may be many such structures and the mechanically driven hysteresis will be a series of transitions between them. In the course of these transitions, some external flux -- with accompanying energy -- must occur, and the energy to create these transition structures must be furnished by the mechanical stress field and will be largely lost when the external energy is reduced as the domains approach a new ideal configuration. This loss is intrinsic to the domain structure and cannot be eliminated except by the temperatures that eliminate magnetism all together. 
A schematic of two such ideal domain structures is shown in Fig. 2, which is only a twodimensional simplification of real three-dimensional domain structures. We see here just the cases $\mathrm{N}=$ 5 and $N=6$, but we can see that the two structures are similar except for edge effects. If we imagine that $N=1,000$, say, then there would be many similar structures with slightly different proportions of the two magnetic orientations, which have correspondingly oriented magnetostriction, and an applied stress could then force transitions between these geometrically similar states and an oscillating stress would propel the system through a repeating succession of self-similar states, leading to self-similar losses, as observed. Note that this mechanism is a form of topological hysteresis, in which the initial and final states are similar, and so might be considered topologically very close, but the transition states are not. We see in hindsight that the experiments of Porteseil et al. separated the topological hysteresis from the force-field hysteresis.
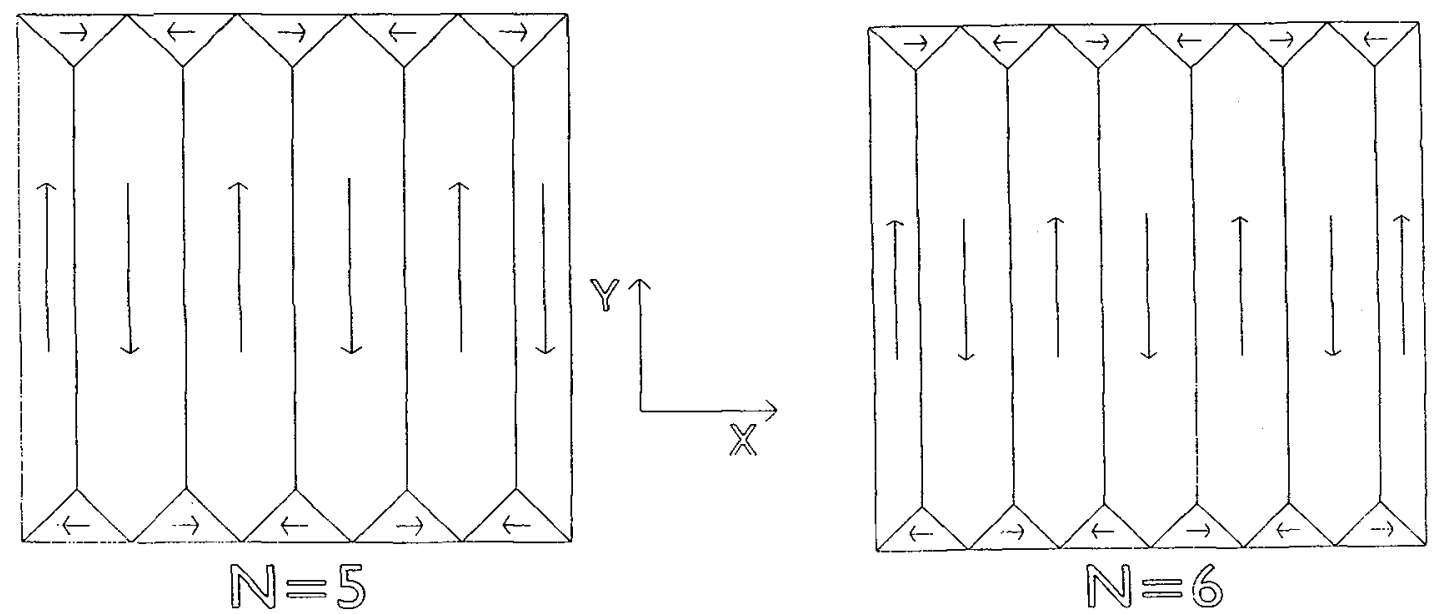

Figure 2: Simple diagram of a model for MMD. Two possible domain configurations for a body. Each configuration has zero magnetization and is metastable, but a transition between the two requires extra energy and must be activated by an external influence, such as field, stress or temperature. The arrows show the direction of magnetization in each domain.

\section{References}

[1] Smith, G.W., Birchak, J.R., J. Appl. Phys. 39 (1968) 2311-2316.

[2] Smith, G.W., Birchak, J.R., J. Appl. Phys. 40 (1969) 5174-5178.

[3] Coronel, V.F., Beshers, D.N., J. Appl. Phys. 64 (1988) 2006-2015.

[4] Beshers, D.N., Materials Science Forum 119-121 (1992) 17-28.

[5] Beshers, D.N., "Internal Stress: measures by mobility of Bloch walls and dislocations",

Micromechanics of Advanced Materials, Cleveland 30 October - 2 November 1995, S.N.G. Chu, et al. Eds. (The Minerals, Metals \& Materials Society, Warrendale, PA, 1995) pp. 17-23.

[6] Vergne, R., Porteseil, J.L., Cotillard, J.C., J. Magn. Magn. Mater.15-18 (1980) 1470-1472.

[7] Hilzinger, H.R., Phil. Mag. 36 (1977) 225-234.

[8] Guberman, H.D., Beshers, D.N., Acta Met. 16 (1968) 167-176.

[9] Govaer, D., Beshers, D.N., J. Appl. Phys. 63 (1988) 2358-2363.

[10] Smith, G.W., Birchak, J.R., J. Appl. Phys. 41 (1970) 3315-3321.

[11] Birchak, J.R., Smith, G.W., J. Appl. Phys. 43 (1972) 1238-1245.

[12] Porteseil, J.L., Cotillard, J.C., Vergne, R., Ferrari, G., J. de Phys. 42 (1981) 1253-1261. 